\title{
Monoclonal Antibody and its Diagnostic Application- Review
}

\author{
Tigabu Demlie ${ }^{1}$, Endale Balcha ${ }^{2}$ and Haben Fesseha ${ }^{3 *}$ \\ ${ }^{1}$ College of Veterinary Medicine, Wollo University, Dessie, Ethiopia \\ ${ }^{2}$ College of Veterinary Science, Mekelle University, Mekelle, Ethiopia \\ ${ }^{3}$ School of Veterinary Medicine, Wolaita Sodo University, Wolaita Sodo, Ethiopia \\ *Corresponding author: Haben Fesseha, School of Veterinary Medicine, Wolaita Sodo University, P.O.Box 138, Wolaita \\ Sodo, Ethiopia
}

\section{ARTICLE INFO}

Received: 幽 September 07, 2020

Published: 歯 September 24, 2020

Citation: Tigabu Demlie, Endale Balcha, Haben Fesseha. Monoclonal Antibody and its Diagnostic Application- Review. Biomed J Sci \& Tech Res 30(4)-2020. BJSTR. MS.ID.004997.

Keywords: Enzyme-Linked Immunosorbent Assay;Hybridoma Technology;Immunohistochemistry; Monoclonal-Antibodies; Western Blot

\begin{abstract}
Monoclonal antibodies are immunoglobulins which have great advantages over the field of biochemistry, immunology, and biotechnology and also known as mouse antibodies because they are produced by mouse. As they are difficult to produce in contrast to polyclonal antibodies, they are precious (expensive) than polyclonal. They are also produced by hybridoma technology which was discovered by Georges Kohler and Cesar Milstein by first immunize the mouse, then extract the plasma cells from the spleen and fused with myeloma cells (cancer cells), finally produce hybridoma cells. During vaccination (immunization) of mice, animal welfare is taken into consideration. They have prominent applications in diagnosis, so monoclonal antibodies are incorporated in diagnostic techniques (Western immunoblotting,ELISA, Immunofluorescence test, and immunohistochemistry) in the diagnosis of livestock diseases. Monoclonal antibodies in diagnostic tests are important for detecting an antigen or antibody against microbes.
\end{abstract}

\section{Introduction}

An antibody, also known as an immunoglobulin [1], is a large, Y-shaped protein produced mainly by plasma cells that is used by the immune system to neutralize pathogens such as pathogenic bacteria and viruses. The antibody recognizes a unique molecule of the pathogen, called an antigen, via Fab's variable region [2]. Each tip of the "Y" of an antibody contains a paratope that is specific for one particular epitope on an antigen. Based on affinity antibodies are classified; one as Monoclonal antibodies are antibodies that are made by identical immune cells that are all clones of a unique parent cell. Monoclonal antibodies can have monovalent affinity, in that they bind to the same epitope, and the other is polyclonal antibodies bind to multiple epitopes (https://en.wikipedia.org/ wiki/Monoclonal_antibody). Monoclonal antibodies are also produced by an old immunological technique with great applications in the fields of biochemistry, immunology, and biotechnology among others [3]. The production of monoclonal antibody using hybridoma technology was first invented by Georges Kohler and Cesar Milstein [4].

One unique Merit of hybridoma production is that a mixture of antigens can be used to generate specific antibodies. Additionally, this also enables screening of antibodies of choice from a mixture of antibody populations generated by purified antigen where single-cell clones can be isolated [5]. MABs can be used in immunodiagnostic techniques as reagents to identify the antigen of the causative agents or indirectly for serological detection of antibodies against the causative agents [6,7]. They have also been reported to be used in experimental purposes ranging from molecular detection of antigenic epitopes to monoclonal antiidiotype antibody for utilization as a vaccine to induce protective immunity [8]. Development in biotechnology has contributed to the large scale production of mAbs which forms an integral part of many diagnostic techniques. These assays are frequently employed 
either for the detection of infectious agents or any of its structural components or even the antibodies generated against the infectious agents [9].

An additional role of monoclonal antibodies in disease prediction and detection is promising. Monoclonal antibody technology plays a significant role in the development of specific serologic reagents towards antigen in limited amounts. They provide both highly specific and reproducible immunological assay for rapid and accurate diagnosis of different types of infectious diseases [10]. The advantages and disadvantages of the use of monoclonal antibodies in several immunoassays need to be evaluated. This will help in the specific diagnosis of infectious diseases in different laboratories [11]. Thus, this review aims to highlight monoclonal antibodies and their Diagnostic applications including their Production technology.

\section{Monoclonal Antibodies}

\section{Structure and Function}

Antibodies are large heterodimeric molecules and are composed of two types of polypeptide chains, called the heavy and the light chain. The two types of light chains are kappa and lambda. By cleavage with the enzyme papain, the fragment-antigen binding part can be separated from the fragment constant part of the molecule. The Fab fragments contain the variable domains, which consist of three antibody hypervariable amino acid domains responsible for the antibody specificity embedded into constant regions [12]. Antibodies are a key component of the adaptive immune response, playing a central role both in the recognition of foreign antigens and the stimulation of an immune response to them. The advent of monoclonal antibody technology has made it possible to raise antibodies against specific antigens presented on the surfaces of tumors. Monoclonal antibodies can be acquired in the immune system through passive immunity or active immunity. The merits of active monoclonal antibody therapy are the fact that the immune system will produce antibodies long-term, with only a short-term drug administration to induce this response. However, the immune response to certain antigens may be inadequate, especially in the elderly. Additionally, adverse reactions from these antibodies may occur because of long-lasting responses to antigen. Passive monoclonal antibody therapy can ensure consistent antibody concentration and can control for adverse reactions by stopping administration. However, repeated administration and consequently higher costs for this therapy are major disadvantages [13].

\section{Production of Monoclonal Antibody}

Hybridoma Technology: Hybridoma technology a technique for producing large numbers of Mouse antibodies and was first invented by G.Kohler and C.Milstein during 1975 and was awarded the Nobel Prize along with N. Jeme in the Physiology and Medicine field during 1984 [14]. This technology is essential to produce a hybrid cell. These hybrid cells are produced by fusing B-lymphocyte with tumor cell and they are called myeloma cells. As the result, these hybrid Cells produced from hybridoma technology are cultured in a laboratory and passaged using a mouse peritoneal cavity and these cells produce monoclonal antibodies, and this technology is known as hybridoma technology (https://www.biotecharticles.com). To produce monoclonal antibodies by Hybridoma technology; mice are first exposed to the antigen that an antibody is to be generated against. Usually, this is made by a series of injections of the antigen in question. These injections are typically followed by the use of in vivo electroporation, which significantly enhances the immune response. Once splenocytes are isolated from the mammal's spleen, the B cells are fused with immortalized myeloma cells. The fusion of the B cells with myeloma cells can be made using electrofusion. Electrofusion causes the B cells and myeloma cells to align and fuse with the application of an electric field. Alternatively, the B-cells and myelomas can be made to fuse by chemical protocols, most of them use polyethylene glycol. The myeloma cells are selected beforehand to ensure they are not secreting antibodies themselves and that they lack the hypoxanthine-guanine phosphoribosyl transferase gene, making them sensitive to the HAT medium (https://en.wikipedia. org/wiki/Hybridoma_technology).

Fused cells are incubated in the HAT medium for about $10-14$ days. Aminopterin blocks the pathway that allows for nucleotide synthesis. Hence, unfused myeloma cells die, as they cannot produce nucleotides by the de novo or salvage pathways because they lack HGPRT. Avoidance of the unfused myeloma cells is crucial because they have the potential to outgrow other cells, especially weakly established hybridomas. Unmixed B cells die as they have a short life span. For this reason, only the B cell-myeloma hybrids survive, since the HGPRT gene coming from the B cells is functional. These cells produce antibodies and are immortal. The incubated medium is then diluted into multi-well plates to such an extent that each well contains only one cell. Since the antibodies in a well are produced by the same B cell, they will be directed towards the same epitope, are thus monoclonal antibodies (https://en.wikipedia. org/wiki/Hybridoma_technology\#Applications).

The next stage is a rapid primary screening process, which identifies and selects only those hybridomas that produce antibodies of proper specificity. The first screening technique used is called ELISA. The hybridoma culture supernatant, secondary enzyme-labeled conjugate, and the chromogenic substrate is then incubated, and the formation of a colored product indicates a Positive hybridoma. Alternatively, immunocytochemical screening can also be used [15]. The B cell that produces the desired antibodies can be cloned to produce many identical daughter clones. Supplemental media containing interleukin- 6 are essential for this step. Once a hybridoma colony is established, it will continually grow in culture medium and produce antibodies [16]. Multiwell plates are used initially to grow the hybridomas, and after selection, are changed to larger tissue culture flasks. This maintains the well-being of the 
hybridomas and provides enough cells for cryopreservation and supernatant for subsequent investigations. The culture supernatant can produce 1 to $60 \mu \mathrm{g} / \mathrm{ml}$ of the monoclonal antibody, which is maintained at $-20^{\circ} \mathrm{C}$ or lower temperature [17]. By using culture supernatant or a purified immunoglobulin preparation, further analysis of a potential monoclonal antibody-producing hybridoma can be made in terms of reactivity, specificity, and cross-reactivity [18].

Identification and Isolation of the Hybridoma Cells: Hybridoma cells producing specific antibodies for the antigen used to immunize the mouse are identified and isolated by the following method (https://www.biotecharticles.com/Others-Article/ Hybridoma-Technology-A-Biotechnology-Technique-378.html). The first step is specific antibodies present in each microwell are identified using one of the methods such as precipitation method or agglutination method. The most commonly used and most sensitive and rapid method is ELISA (https://www.biotecharticles. com/OthersArticle/Hybridoma-Technology-A-BiotechnologyTechnique-378.html). Secondly, the Wells which contain the antibodies specific to the antigens are identified and hybridoma cells are isolated from these wells and cultured (cloned). This ensures that these hybridoma cells can produce the same single type of antibodies specific to the antigen used (https://www. biotecharticles. com/Others Article/ Hybridoma Technology A Biotechnology-Technique-378.html). Finally, these hybridoma cells are multiplied using in vitro (in a laboratory) or in vivo (in mouse) method and Mass Production of monoclonal Antibodies (https:// www.biotech articles.com/Others Article/ Hybridoma Technology A Biotechnology-Technique-378. html).

Laboratory animal ethical considerations: Increasingly use of laboratory animals in the scientific Experiment has drawn more attention to the first ethics of these valuable creatures. There are international guidelines for the use and care of animals in scientific procedures. One of these guidelines is represented by the National Advisory Committee for Laboratory Animal Research, which seems to launch concise yet comprehensive considerations about the use and care of animals for scientific and research purposes [19] These international guidelines are classified into three parts that should be considered together as a comprehensive document: The first part, "Guiding principles for the care and use of animals for scientific purposes", explains all principles which promote the humane and responsible care and use of animals for research and scientific goals. The concept of the principles describes the 3Rs-Replacement, Reduction, and Refinement [20]. The second part, "Guidelines for institutional animal care and use committee", includes the guiding principles for using and care of animals for research goals and explains in detail the operational aspects about the Institutional Animal Care and Use Committee. The third part, "Training guidelines", describes the training activities and requirements for users of animals and animal facilities personnel. This includes the scope of the core curriculum and the relevant core competencies, such as specific workshops for animal procedures [21-24].

\section{Diagnostic Application of Monoclonal Antibody}

The importance of monoclonal antibodies is numerous and includes the prevention, diagnosis, and treatment of disease (https://en.wikipedia.org/wiki /Hybridoma_ technology \#Applications). The application of monoclonal antibodies in diagnosis is by far the most advanced, especially for tests that are performed on body fluids such as blood and urine [25]. Monoclonal antibodies can be used to detect the presence of antigens. They can also be used in different techniques which include ELISA, western blot, immunoblot blot, flow cytometry, immunohistochemistry, radioimmunology assay, electron microscopy, fluorescence microscopy and confocal microscopy [26-29]. Additionally, monoclonal antibodies are used for the detection of antigen as well as antibodies against bacterial diseases of livestock; for detection of antigen as well as antibodies against fungal diseases of livestock; for detection of antigen as well as antibodies against viral diseases of livestock and diagnosis of parasitic diseases (https://pdfs. semanticscholar.org/937c.pdf).

\section{Western Immunoblotting}

The inception of the protocol for protein transfer from an electrophoresis gel to a membrane, protein blotting has evolved greatly. Western blotting analysis can detect one protein in a solution that contains any number of proteins and giving the protein information, and it is widely used in protein detection. It is a method in molecular biology or biochemistry or immunogenetics to detect protein in a given sample of tissue homogenate or extract, which is normally used with a high antibody directed against the desired antigen [30]. No doubt, western blotting has been an invaluable tool used in the detection of viruses. Novel western blotting was developed based on monoclonal antibody for the detection of Cytomegalovirus in latently infected blood donors, pregnant women, and transplant recipients with ongoing human cytomegalovirus. Evaluation of this assay revealed high sensitivity and specificity compared to the conventional IgM based ELISA [31]. Western blotting can produce qualitative and semi-quantitative data about the protein of interest. It is an important technique used in cell and molecular biology. It enables the researchers to identify the specific protein from a mixture of proteins extracted from cells as well as the evaluation of their size and amount. The SDS PAGE technique is a prerequisite for western blotting (https:// microbeonline.com/western-blot technique principle procedures advantage and disadvantages).

Western blotting also known as protein blotting or immunoblotting is a rapid and sensitive assay for the detection and charac- 
terization of proteins. It is based on the principle of immunochromatography where proteins are separated into polyacrylamide gel according to their molecular weight (https://microbeonline.com/ westernblottechniqueprincipleproceduresadvantageanddisadvantages). The proteins thus separated by PAGE are then transferred or electro transferred onto nitrocellulose membrane and are detected using a specific primary antibody and secondary enzyme-labeled antibody and substrate (https://microbeonline.com/westernblottechniqueprincipleproceduresadvantageand disadvantages).

\section{Monoclonal antibody in Immunohistochemistry}

Immunohistochemistry is one of the diagnostic methods which involve the process of selectively imaging antigens in cells of a tissue section by exploiting the principle of antibodies binding specifically to antigens in biological tissues [32]. Antibody-based applications depend on the specific binding of an antibody to the target epitope to generate accurate expression data. Blocking reactive epitopes and endogenous enzymes before primary antibody incubation on specific binding and mitigates false positive error (https://www. novusbio.com/blocking-non-specific-binding). To block nonspecific binding, the most Common buffers to block non-specific bindings are normal serum, such as BSA. If blocking with normal serum, the species of the animal serum is dependent on the host of the secondary antibody. For example, use goat serum if using a goat anti-mouse secondary. However, the choice of blocking buffer is contingent on the antigen type and type of detection used. For instance, if an alkaline-phosphatase conjugated secondary antibody is used, then the blocking serum should be diluted in TBS. PBS will interfere with the alkaline phosphatase (https://www.novusbio. com/blocking-non-specific-binding).

In order to determine the non-specific signal in immunohistochemistry experiment, a negative tissue control should be run with each immunohistochemistry experiment to determine the amount of background staining. The presence of non-specific staining will be associated with the deposition of chromogenic or fluorescent signals in the negative tissue control sample (https:// www.novusbio.com/blocking-non-specific-binding). After blocking non-specific binding, Immunohistochemical staining is widely used in the diagnosis of abnormal cells such as those found in cancerous tumors. Specific molecular markers are characteristic of particular cellular events such as apoptosis. Immunohistochemistry is also widely used in basic research to understand the distribution and localization of biomarkers and differentially expressed proteins in different parts of biological tissue. Visualizing an antibodyantigen interaction can be performed in several ways. In the most common instance, an antibody is conjugated to an enzyme, such as peroxidase, that can catalyze a color-producing reaction. Alternatively, the antibody can also be tagged to a fluorophore, such as fluorescein [33].

Immunofluorescence Test: Immunofluorescence is a technique used for light microscopy with a fluorescence microscope and is used primarily on microbiological samples. This method uses the specificity of antibodies to their antigen to target fluorescent dyes to specific biomolecule targets within a cell, and therefore allows visualization of the distribution of the target molecule through the sample. The specific region an antibody recognizes on an antigen is called an epitope [34]. There have been efforts in epitope mapping since many antibodies can bind the same epitope and levels of binding between antibodies that recognize the same epitope can vary [35]. Additionally, Immunofluorescence can also be used on tissue sections, cultured cell lines, and may be used to analyze the distribution of proteins, glycans, and small biological and non-biological molecules. This technique can even be used to visualize structures such as intermediate-sized filaments (Franke et al, 1978). If the topology of a cell membrane has yet to be determined, epitope insertion into proteins can be used in conjunction with immunofluorescence to determine structures [36].

To make fluorochrome-labeled antibodies, a fluorochrome must be conjugated to the antibody. Likewise, an antigen can also be conjugated to the antibody with a fluorescent probe in a technique called fluorescent antigen technique. Staining procedures can apply to both fixed antigens in the cytoplasm or to cell surface antigens on live cells, called "membrane immunofluorescence". It is also possible to label the complement of the antibody-antigen complex with a fluorescent probe. In addition to the element to which fluorescence probes are attached, there are two general classes of immunofluorescence techniques: primary and secondary. The following explanation will focus primarily on these classes in terms of conjugated antibodies [37]. The first class is Primary immunofluorescence uses a one, primary antibody, chemically linked to a fluorophore. The primary antibody recognizes the target antigen and binds to a specific region called the epitope. The attached fluorophore can be detected via fluorescent microscopy; will emit a specific wavelength of light once excited. Direct immunofluorescence, although somewhat less common, has notable advantages over the secondary procedure. The direct attachment of the messenger to the antibody reduces the number of steps in the procedure, saving time, and reducing non-specific background signals [38].

The second class also called Secondary immunofluorescence uses two antibodies; the unlabeled first antibody specifically binds the target molecule, and the secondary antibody, which carries the fluorophore, recognizes the primary antibody, and binds to it. Multiple secondary antibodies can bind a single primary antibody. This provides signal amplification by increasing the number of fluorophore molecules per antigen. This protocol is more complex and time-consuming than the primary (or direct) protocol above but allows more flexibility because a variety of different secondary antibodies and detection techniques can be used for a given primary antibody. 


\section{Monoclonal Antibodies in Diagnostic Histopathology}

In histopathology by the help of monoclonal antibodies, tissues and organs can be classified based on their expression of certain defined markers, which reflect tissue or cellular genesis. Prostatespecific antigen, placental alkaline phosphatase, human chorionic gonadotrophin, $\alpha$-fetoprotein, and others are organ-associated antigens and the production of monoclonal antibodies against these antigens helps in determining the nature of a primary tumor. Monoclonal antibodies are especially useful in distinguishing morphologically similar lesions, like pleural and peritoneal mesothelioma, adenocarcinoma, and in the determination of the organ or tissue origin of undifferentiated metastases. Selected monoclonal antibodies help in the detection of occult metastases by immuno-cytological analysis of bone marrow, other tissue aspirates, as well as lymph nodes and other tissues and can have increased sensitivity over normal histopathological staining.

\section{Enzyme-linked Immunosorbent Assay}

Many immunological assays have been developed for the diagnosis of infectious diseases which were either through the detection of antigen or anti-antibodies in sera of infected animals. In this regard, specific epitopes targeted by the produced monoclonal antibodies were protein in nature. This approach uses mAbs, so they were used in a blocking ELISA to detect the antigen of the parasite. The mAbs have shown some reactions to the homologous antigen in an indirect ELISA but did not reveal positivity with the SDS-PAGE separated homologous antigen in a Western blot analysis, suggesting conformational epitope specificity. The mAbs were filariform stage-specific and thus could not also detect the antigen of the rhabditiform larvae contained in the patient's stool. Due to the predominantly zoonotic importance of most nematode parasites, efficient control measures can only be achieved if accurate diagnosis of these conditions can be made. A monoclonal antibody specific for Toxocara cati was prepared and was experimentally used to increase the sensitivity of ELISA for antigen detection. IgG3 isotype was produced which has shown a good reaction against the excretory-secretory antigen of the larval stage of T. cati. The antibody shows no cross-reaction with the antigens of other nematode parasites and had enough sensitivity to detect circulating antigens in serum. The results indicated that this antigen detection system provides a useful method for the diagnosis of both visceral and ocular larva migrans caused by T. cati and fulfills the requirement of a serological assay for the diagnosis of toxocariasis.

In this way, specific monoclonal antibodies have been produced to further characterize the suppressive components of the extract of Ascaris suum. The immunosuppressive fractions isolated from the adult worms were used to stimulate antibody production. The proteins PAS-1, PAS-2, and PAS-3 were purified from the crude extract and were prepared as antigen with ovalbumin for the immunization of mice. Three monoclonal antibodies MAIP-1, MAIP-
2, and MAIP-3 obtained from the cloned hybrid cells were screened to determine their specificities in ELISA using coated plates with each fraction of the A. suum extract isolated by gel filtration. They were shown to react with different antigenic epitopes of high molecular weight proteins. These antibodies have recognized the antigen with different affinity constants. Cryptococcosis is a fungal disease of major concern and potentially fatal disease that is the cause of the most common life-threatening meningitis in patients with acquired immunodeficiency syndrome. Due to the difficulties and lack of sensitivity and specificity associated with the conventional microbiological and histopathological diagnostic techniques of fungal diseases, interest in consideration of developing non-culture approaches arose. The recent birth of the use of monoclonal antibody diagnosis plays an important role in early diagnosis for the guide to appropriate treatment and to prevent mortality.

The capsular polysaccharide antigen has been considered as one of the most valuable and rapid tests for fungi. Murex Cryptococcus test incorporated with a mouse monoclonal IgMbased latex agglutination assay has been reported to effectively eliminate false-positive reactions. In order to reduce cross-reaction with other fungal organisms in the detection of histoplasmosis, a $\mathrm{mAb}$ was produced that recognizes a species-specific antigenic epitope of Histoplasma spp by inhibition ELISA. Thus, a more specific detection technique was achieved. In the same vein, was also applied in an inhibition ELISA for the detection of circulating antigen of Paracoccidiodomyces brasiliensis that revealed a more promising method. Competitive ELISA assays based on mAbs not only allow specific detection but also aid accurate quantification of antibodies to human and animal viruses. For example, evaluated the use of mAbs based on the neutralizing epitope of haemagglutinin protein in the titration of antibodies specific to Peste des petits ruminant's virus. They concluded that the ELISA test showed potentials in replacing virus neutralization tests which have been widely used for sero-surveillance, sero-monitoring, diagnosis, and end-point titration of PPR virus antibodies. Similarly, a competitive ELISA assay was found useful for the evaluation of the H5 type influenza virus in samples that are difficult to be evaluated by the hemagglutination inhibition test. In this regard, the assay was found to be very sensitive with excellent diagnostic performance and discriminatory power with high sensitivity and specificity values. Compared with the indirect ELISA, monoclonal antibody-based sandwich direct ELISA was 7 times more sensitive in the detection of FMD virus when Real-Time PCR was used as a gold standard.

\section{Conclusion and Recommendation}

It can be concluded that monoclonal antibody production is a very sophisticated process. It requires immunization of antigens against a specific antigen, collection of spleen cells from immunized mice, and fusing the spleen cells with myeloma cell with an immortal myeloma cell. The technology is referred to as hybridoma technology 
which targets to produce a monoclonal antibody against a specific epitope of antigen as opposed to a polyclonal antibody which is specific to multiple epitopes in an antigen. Monoclonal antibodies have been utilized for diagnostic applications like western blotting, immunohistochemistry, and ELISA. In conclusion, the technology is not widely used in Ethiopia, training with this regard should be intensified. Moreover, it should be used for different diagnostic and therapeutic purposes in our country.

\section{References}

1. Akiyoshi, Kawamura (1983) Immunofluorescence in medical science: with 28 tab. In Akiyoshi, Kawamura (Eds.), Springer ua.

2. Andama AO, den Boon S, Meya D, Cattamanchi A, Worodria W (2013) Prevalence and outcomes of cryptococcal antigenemia in HIVseropositive patients hospitalized for suspected tuberculosis in Uganda. Journal of Acquired Immune Deficiency Syndrome 63(2): 189-194.

3. Baxter, David (2007) Active and passive immunity, vaccine types, excipients, and licensing. Occupational Medicine 57(8): 552-556.

4. Buss NAPS, Henderson SJ, McFarlane M, Shenton JM, De Haan L (2012) Monoclonal antibody therapeutics: History and future. Current Opinion in Pharmacology 12(5): 615-622.

5. Chowdhury N, Sood NK, Lal S, Gupta K, Singla LD (2013) Development of some larval nematodes in experimental and natural animal hosts: An insight into development of pathological lesions vis-a-vis host-parasite interactions. The Scientific World Journal Article ID 162538, 8 pages.

6. Deb R, Chakraborty S, Veeregowda B, Verma AK, Tiwari R (2013) Monoclonal antibody and its use in the diagnosis of livestock diseases. Special Issue in Antibody Research 4(4A): 50-62.

7. FrankeWW, Schmid E, Osborn M, Weber K (1978) Different intermediatesized filaments distinguished by immunofluorescence microscopy. Proceedings of the National Academy of Sciences of the United States of America 75(10): 5034-5038.

8. Fritschy J, Härtig W (2001) Immunofluorescence. ELS.

9. Ghosh S, Ansar W (2013) Monoclonal Antibodies: A tool in clinical research. Indian Journal of Clinical Medicine 4: 9-21.

10. Gomez BL, Figueroa JI, Hamilton AJ, Diez S, Rojas M (1999) Detection of the 70-kilodalton Histoplasma capsulatum antigen in serum of histoplasmosis patients: Correlation between antigenemia and therapy during follow-up. Journal of Clinical Microbiology 37(3): 675-680

11. Gomez BL, Figueroa JI, Hamilton AJ, Diez S, Rojas M (1998) Antigenemia in patients with paracoccidioidomycosis: Detection of the 87-kilodalton determinant during and after antifungal therapy. Journal of Clinical Microbiology 36 (11): 3309-3316.

12. Gupta SK, Singla LD (2012) Diagnostic trends in parasitic diseases of animals. In: Veterinary Diagnostics: Current Trends (Gupta RP, Garg SR, Nehra V, Lather D, editors), Satish Serial Publishing House, Delhi, India, pp. 81-112.

13. Hau J, Van Hoosier GL (2003) Animal research ethics. In Olsson IAS, Robinson P, Pritchett K, Sandoe P (Eds.), Handbook of laboratory animal science. Boca Raton: CRC Press pp. 13-31.

14. https://en.wikipedia.org/wiki/Hybridoma_technology

15. https://en.wikipedia.org/wiki/Monoclonal_antibody

16. https://microbeonline.com/westernblottechnique $\% 20$ principle $\% 20$ procedures $\% 20$ advantage $\% 20$ and $\% 20$ disadvantages /) :\% 20 Accessed\%20on\%20June\%20

17. (2018) https://www.biotecharticles.com/Others-Article/HybridomaTechnology-A-Biotechnology-Technique-378.html.
18. (2018) https://www.novusbio.com/blocking-non-specific-binding.

19. Hubrecht R, Kirkwood J (2010) Implementing the three Rs in research using animals. In Richmond J (Eds.), UFAW handbook on the care and management of laboratory animals. Blackwell publishing, London 2: 2337.

20. Janeway CA Jr, Travers P, Walport M, Shlomchik MJ (2001) Immunobiology ( $5^{\text {th }}$ edn.), Garland Publishing, USA ISBN 0-8153-3642-X.

21. Kiska DL, Orkiszewski DR, Howell D, Gilligan PH (1994) Evaluation of new monoclonal antibody-based latex agglutination test for detection of cryptococcal polysaccharide antigen in serum and cerebrospinal fluid. Journal of Clinical Microbiology 32(9): 2309-2311.

22. Lazzarotto T, Maine GT, Dal Monte P, Ripalti A, Landini MP (1997) A novel western blot test containing both viral and recombinant proteins for anticytomegalovirus immunoglobulin M detection. Journal of Clinical Microbiology 35(2): 393-397.

23. Mandrell RE, Griffiss JM, Macher BA (1988) Lipooligosaccharides (LOS) of Neisseria gonorrhoeae and Neisseria meningitidis have components that are immunochemically similar to precursors of human blood group antigens. Carbohydrate sequence specificity of the mouse monoclonal antibodies that recognize crossreacting antigens on LOS and human erythrocytes. Journal of Experimental Medicine 168(1): 107-126.

24. Marra NM, Chiuso Minicucci F, Machado GC, Zorzella Pezavento SFG, França TGD (2010) Faecal examination and PCR to detect Strongyloides venezuelensis in experimentally infected Lewis rats. Memoriaz Instituto Oswaldo Cruz 105(1): 57-61.

25. Moreno A, Lelli D, Brocchi E, Sozzi E, Vinco LJ (2013) Monoclonal antibody-based ELISA for detection of antibodies against H5 avian influenza viruses. Journal of Virological Methods 187(2): 424-430.

26. Morioka K, Fukai K, Sakamoto K, Yoshida K, Kanno T (2014) Evaluation of Monoclonal Antibody-Based Sandwich Direct ELISA (MSD-ELISA) for Antigen Detection of Foot-and-Mouth Disease Virus Using Clinical Samples. PLoS One 9(4): e94143.

27. Nelson PN, Reynolds GM, Waldron EE, Ward E, Giannopoulos K (2000) Demystified Monoclonal antibodies. Molecular pathology: MP 53(3): 111-117.

28. Oshiro TM, Rafael A, Enobe CS, Fernandes I, Macedo Soares MF (2004) Comparison of different monoclonal antibodies against immunosuppressive proteins of Ascaris sum. Brazilian Journal of Medical and Biological Research 37(2): 223-226.

29. Pal P, Dowd KA, Brien JD, Edeling MA, Gorlatov S (2013) Development of a Highly Protective Combination Monoclonal Antibody Therapy against Chikungunya Virus. PLoS Pathogens 9(4): e1003312

30. Pandey S (2010) Hybridoma technology for production of monoclonal antibodies. International Journal of Pharmaceutical Sciences Review and Research 1(2): 88-94.

31. Rhoades RA, Pflanzer RG (2002) Human Physiology. In Rhoades RA, Pflanzer RG (Eds.), (4 ${ }^{\text {th }}$ ed.), Thomson Learning. pp. 584.

32. Singh RP, Sreenivasa BP, Dhar P, Shah LC, Bandvopadhyay SK (2004) Development of a monoclonal antibody-based competitive-ELISA for detection and titration of antibodies to peste des petits ruminants (PPR) virus. Veterinary Microbiology 98(1): 3-15.

33. Smith BT (2012) Introduction to Diagnostic and Therapeutic. University of New Mexico Health Science Center 17(0039): 1-34.

34. Taweethavonsawat P, Chaicumpa W, Chaisri U, ChuenbalU, Sakolvaree Y (2002) Specific monoclonal antibodies to Strongyloides stercoralis: A potential diagnostic reagent for strongyloidiasis. Asian Pacific Journal of Allergy and Immunology 20(4): 247-256.

35. Wang Honggang, Lee Eun Woo, Cai Xiaokun, Ni Zhanglin, Zhou Lin (2008) Membrane Topology of the Human Breast Cancer Resistance Protein (BCRP/ABCG2) Determined by Epitope Insertion and Immunofluorescence. Biochemistry 47(52): 13778-13787. 
36. Wilson K, Walker J (2010) Principles and Techniques of Biochemistry and Molecular Biology. Director, 7: 802.

37. Yang Y, Ma H (2009) Western Blotting and ELISA Techniques. Researcher $1(2): 67-86$.

\section{ISSN: 2574-1241}

DOI: $10.26717 /$ BJSTR.2020.30.004997

Haben Fesseha. Biomed J Sci \& Tech Res

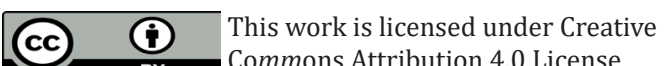

Submission Link: https://biomedres.us/submit-manuscript.php
38. Yeo SF, Wong B (2002) Current status of nonculture methods for diagnosis of invasive fungal infections. Clinical Microbiology Reviews 15(3): 465-484.

$\begin{array}{ll}\text { BIOMEDICAL } & \text { Assets of Publishing with us } \\ \text { RESEARCHES } & \text { - Global archiving of articles } \\ & \text { - Immediate, unrestricted online access } \\ & \text { - Rigorous Peer Review Process } \\ & \text { - Anttps://biomedres.us/ }\end{array}$

